\title{
Role of Structured Education in Reducing Lypodistrophy and its Metabolic Complications in Insulin-Treated People with Type 2 Diabetes: A Randomized Multicenter Case-Control Study
}

\author{
Sandro Gentile (D) - Giuseppina Guarino • Teresa Della Corte • Giampiero Marino • Ersilia Satta • \\ Maria Pasquarella - Carmine Romano - Carmelo Alfrone · Felice Strollo on behalf of AMD-OSDI Study Group \\ on Injection Technique, Nefrocenter Research and Nyx Start-Up
}

Received: December 20, 2020 / Accepted: January 15, 2021 / Published online: March 18, 2021

(c) The Author(s) 2021

\section{ABSTRACT}

Introduction: It is essential to use the correct injection technique (IT) to avoid skin complications such as lipohypertrophy (LH), local inflammation, bruising, and consequent repeated unexplained hypoglycemia episodes (hypos) as well as high HbA1c (glycated hemoglobin) levels, glycemic variability (GV), and insulin doses. Structured education plays a

Lists of all members of the AMD-OSDI Study Group on Injection Technique and the Nefrocenter Research and Nyx Start-Up Study Group are available in the "Acknowledgements" section.

AMD: Italian Association of Diabetes Specialists; OSDI: Italian Diabetes Health Workers Association.

Supplementary Information The online version contains supplementary material available at https:// doi.org/10.1007/s13300-021-01006-0.

S. Gentile $(\bowtie) \cdot$ G. Guarino · T. Della Corte .

G. Marino

Department of Internal Medicine, Campania

University "Luigi Vanvitelli", Naples, Italy

e-mail: s.gentile1949@gmail.com

F. Strollo

Endocrinology and Diabetes, IRCCS San Raffaele

Pisana, Rome, Italy

S. Gentile · G. Guarino - T. Della Corte · G. Marino

E. Satta · M. Pasquarella - C. Romano - C. Alfrone ·

F. Strollo

Nefrocenter Research Network and Nyx Start-Up,

Naples, Italy prominent role in injection technique improvement. The aim was to assess the ability of structured education to reduce (i) GV and hypos, (ii) HbA1c levels, (iii) insulin daily doses, and (iv) overall healthcare-related costs in outpatients with T2DM who were erroneously injecting insulin into LH.

Methods: 318 patients aged 19-75 years who had been diagnosed with T2DM for at least 5 years, were being treated with insulin, were routinely followed by a private network of healthcare centers, and who had easily seen and palpable LH nodules were included in the study. At the beginning of the 6-month run-in period $(\mathrm{T}-6)$, all patients were trained to perform structured self-monitoring of blood glucose and to monitor symptomatic and severe hypos (SyHs and SeHs, respectively). After that (at T0), the patients were randomly and equally divided into an intervention group who received appropriate IT education (IG) and a control group (CG), and were followed up for six months (until $\mathrm{T}+6$ ). Healthcare cost calculations (including resource utilization, loss of productivity, and more) were carried out based on the average NHS reimbursement price list.

Results: Baseline characteristics were the same for both groups. During follow-up, the intra-LH injection rate for the CG progressively decreased to $59.9 \%(p<0.001)$, a much smaller decrease than seen for the IG $(1.9 \%, p<0.001)$. Only the IG presented significant decreases in HbA1c $(8.2 \pm 1.2 \%$ vs. $6.2 \pm 0.9 \% ; p<0.01)$, 
$\mathrm{GV} \quad(247 \pm 61 \mathrm{mg} / \mathrm{dl} \quad$ vs. $\quad 142 \pm 31 \mathrm{mg} / \mathrm{dl}$; $p<0.01)$, insulin requirement $(-20.7 \%$, $p<0.001$ ), and SeH and SyH prevalence (which dropped dramatically from 16.4 to $0.6 \%$ and from 83.7 to $7.6 \%$, respectively; $p<0.001$ ). In the IG group only, costs-including those due to the reduced insulin requirement-decreased significantly, especially those relating to SeHs and SyHs, which dropped to $€ 25.8$ and $€ 602.5$, respectively $(p<0.001)$.

Conclusion: Within a 6-month observation period, intensive structured education yielded consistently improved metabolic results and led to sharp decreases in the hypo rate and the insulin requirement. These improvements resulted in a parallel drop in overall healthcare costs, representing a tremendous economic advantage for the NHS. These positive results should encourage institutions to resolve the apparently intractable problem of LH by financially incentivizing healthcare teams to provide patients with intensive structured education on proper injection technique.

Trial Registration: Trial registration no. 118/15.04.2018, approved by the Scientific and Ethics Committee of Campania University "Luigi Vanvitelli," Naples, Italy, and by the institutional review board (IRB Min. no. 9926 dated 05.05.2018).

Keywords: Diabetes; Direct and indirect costs; Education; Glycemic variability; Hypoglycemia; Lipohypertrophy

\section{Key Summary Points}

\section{Why carry out this study?}

Incorrect injection technique is a widespread phenomenon that can lead to skin lipohypertrophy (LH) in insulintreated subjects with diabetes mellitus (DM).

LH lesions cause significant clinical problems that increase healthcare costs, including those due to glucose variability, hypoglycemic events, and excess insulin utilization.
The aim of the study was to assess the ability of a 6-month intensive, structured patient education program to prevent poor injection habits and related complications and costs in a large group of outpatients with type $2 \mathrm{DM}$.

\section{What was learned from the study?}

Intensive education led to consistently improved metabolic results, a lower insulin requirement, and-for the first time-decreased overall healthcare costs in the target population.

Such positive effects should encourage institutions to resolve the apparently intractable problem of LH by providing healthcare teams with economic incentives to carry out intensive, specialized patient education on correct injection technique.

\section{DIGITAL FEATURES}

This article is published with digital features, including a summary slide, to facilitate understanding of the article. To view digital features for this article go to https://doi.org/10.6084/ m9.figshare.13574384.

\section{INTRODUCTION}

Several literature reports published since the 1980s have suggested that injection technique (IT) is as crucial to achieving glycemic control as the type and dose of insulin delivered [1-6].

Injection technique encompasses a range of procedures that are intended to facilitate the most consistent and least painful delivery of insulin into the subcutaneous tissue. It includes considerations such as injection site and needle length selection, the angle of needle insertion, and skin fold lifting. The injection technique applied can significantly influence the pharmacokinetics (PK) and pharmacodynamics (PD) of insulin. Hence, correct technique helps avoid 
skin complications such as lipohypertrophy (LH), local inflammation, and bruising, as well as metabolic consequences, including repeated episodes of unexplained hypoglycemia (hypos), high glycemic variability (GV), and high HbA1c levels [7-13]. Injection site rotation across large surfaces, avoidance of repeated needle reuse, and $45^{\circ}$ angling of $>4 \mathrm{~mm}$ long needles into the pinched skin are essential actions to avoid the changes mentioned above and ensure optimal insulin absorption [14-17].

Moreover, therapeutic efforts to keep glycated hemoglobin (HbA1c) within target levels quite often lead to an increased risk for hypoglycemia, especially in insulin-treated patients [18]. Hypos, especially severe ones, have significant clinical, social, and economic impacts [18]. From a clinical standpoint, they can cause a broad set of symptoms ranging from general discomfort to seizures and coma, or even sudden cardiac arrhythmic death or lethal brain damage in the case of long-term glucose deprivation [19]. Therefore, it is crucial to inject insulin correctly to prevent hypos caused by improper injection technique. Notoriously, besides increasing direct and indirect health costs [20], repeated hypos severely affect patient health by increasing cardiovascular and dementia risk [21-24]. Mild symptomatic hypos increase cardiovascular risk and elevate the risk of all-cause hospitalization/mortality, resulting in an excessive economic burden [24, 25]. GV due to improper injection technique is also a major cardiovascular risk factor in type 2 diabetes mellitus (T2DM) patients [26].

Nevertheless, given the high rate of $\mathrm{LH}$ lesions reported in the literature $[5-7,10-13,17]$, it is reasonable to hypothesize that most clinic visits are too brief to allow the patient to be educated in the correct injection technique, even though this is just as crucial to effective diabetes management as discussions about glucose control and dose adjustments [27].

Conversely, health-related quality of life and treatment satisfaction are correlated, and they are affected by a complex interplay between clinical and socioeconomic variables. Some negative impacts on DM patient quality of life are associated with the insulin treatment strategy applied and perceived poor metabolic control [28]. Anecdotal reports point out that proper IT education reduces GV and hypos in LH subjects $[29,30]$. However, to the best of our knowledge, there have been no randomized studies involving sufficiently large patient populations that have specifically addressed this topic.

Based on these premises, we aimed to assess the impact (if any) of providing repeated structured education sessions (SESs) to insulin-treated T2DM patients regarding their diabetes control, and to investigate the effects of a typical improper injection technique as compared to those of delivering a single SES to patients in terms of chronic disease complications and direct/indirect health costs $[20,25,26]$.

\section{METHODS}

This study was designed to be a two-arm, openlabel, multicenter, randomized, case-control study. It was approved by the Ethical and Scientific Committee of the reference center (University "Luigi Vanvitelli" Naples, Italy (Trial Registration no. 118/15.04.2018), and by the Institutional Review Board (IRB Min. no. 9926, dated 05.02.2018). The study was also carried out in accordance with the original Declaration of Helsinki and its subsequent amendments. T2DM patients were enrolled for the study if they had been regularly attending any of the eight diabetes centers (DCs) involved in the study, they met the inclusion criteria, and they gave their informed consent to participate and to have their data published in an anonymous manner.

The primary endpoints of our study were the $\mathrm{GV}$ and the change in hypo rate, and the secondary endpoints were (i) metabolic control as reflected in HbA1c levels, (ii) LH scale, size, and texture, (iii) insulin daily dosage, and (iv) overall healthcare-related costs as a function of intensive educational effort for T2DM patients who were systematically injecting insulin into LH nodules.

All DCs were part of the Nefrocenter Research Network in Southern Italy-a private consortium supported by the National Health 
System in association with Naples University "Luigi Vanvitelli" for several clinical aspects, including the ethics committee. The DCs used the same electronic record system, diagnostic/ therapeutic procedures, and operating standards, adhering to the national program for continuous quality improvement. Their healthcare professionals (HCPs) were well trained to follow the study procedures appropriately.

All patients belonged to a large T2DM group we had just finished screening for LH prevalence [31, 32]. Inclusion criteria were: (1) the presence of insulin injection-related LHs that were evident to sight and palpation (grade I for flat, grade II for protruding) (Fig. 1) [31]; (2) 18-75 years of age; (3) diabetic for at least five years; (4) receiving three to four pen-based insulin analog injections per day (invariably including one long-acting preparation) for the last 12 months at least, with/without add-on oral antihyperglycemic agents; (5) not using add-on hypoglycemic agents; (6) no severe liver disease or cancer; (7) free from dementia or any other functional impairment affecting the patient's ability to adhere to the study protocol; (8) no participation in any other clinical trial for the previous three months; (9) performed selfmonitoring of blood glucose (SMBG) systematically for at least the previous 12 months via data-download-enabled glucose meters; (10) HbA1c level > 5.5\%.

A web-based clinical record form (eCRF) served as a privacy-compliant repository for the following clinical data: age, weight, height, BMI, disease, and insulin treatment duration, daily insulin dose (DID) and number of injections, latest $\mathrm{HbA} 1 \mathrm{c}$ level, latest serum creatinine level, diabetes complications (i.e., cardio/cerebrovascular events, lower limb complications, retinopathy, nephropathy, sensorimotor neuropathy, autonomic neuropathy), chronic noncommunicable diseases including cancer that has been inactive for over 5 years, number

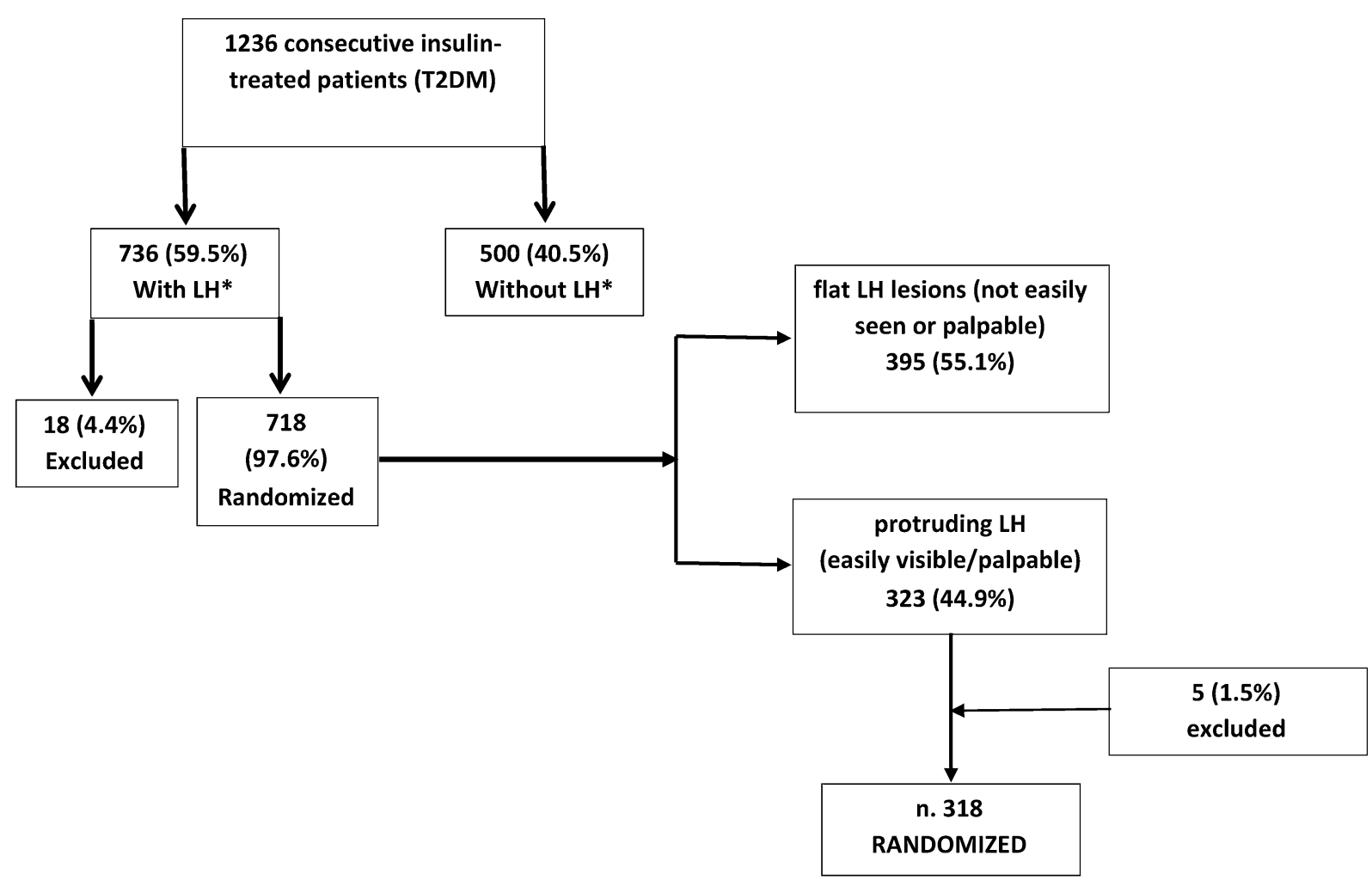

Fig. 1 Flow chart depicting the enrollment procedure for patients who had already been screened for LH lesions during a previous study [31]. LH lipohypertrophy 
of severe or symptomatic hypos (SeHs and SyHs, respectively) experienced during the past 12 months, and GV as previously described [24, 31-33].

Patients were randomized into an intervention group (IG) and a control group (CG) according to a simple centralized randomization system where blinding was achieved by the envelope method.

Glomerular filtration rate (GFR) was calculated according to the Chronic Kidney Disease Epidemiology Collaboration (CKD-Epi) formula. LHs were identified according to a previously reported structured and validated protocol [31-34]. The diagnosis of T2DM was made/confirmed according to the criteria defined by the ADA Standards of Medical Care in Diabetes [35]. The IX International Classification of Diseases, Clinical Modification (ICD9-CM, V82.9 2014) was used to define comorbidities or T2DM-related complications [36].

Hypos were defined as symptomatic or severe based on whether they caused typical symptoms or required help from another person/physician or even hospitalization, as previously described [24, 31-33]. GV was calculated from 21 home-based SMBG results collected at least once a week, where measurements were taken seven times a day (before and $2 \mathrm{~h}$ after each main meal plus $4 \mathrm{~h}$ after dinner) for three days, as previously described [31, 37]. Number and severity of hypos were also discerned from the SMBG results for a series of 3-month intervals (i.e., the $\mathrm{T}-6 / \mathrm{T}-3$ interval, $\mathrm{T}-3 / \mathrm{T} 0$ interval, $\mathrm{T} 0 / \mathrm{T}+3$ interval, and $\mathrm{T}+3 / \mathrm{T}+6$ interval), as confirmed by a validated patient recall-based method [38].

At enrollment, $72 \%$ of the participants used KwikPens with insulin lispro U-100 at mealtime and Abasaglar ${ }^{\circledR}$ (glargine biosimilar) at bedtime. These products are also the least expensive at the national level. For both of these reasons, we prescribed the products at their usual doses to the other participants to avoid biases potentially caused by the use of different pens or insulin preparations. The BD Micro-Fine 4-mm $32 \mathrm{G}$ pen needle (Becton Dickinson, Inc., Franklin Lakes, NJ, USA) was utilized as per new insulin delivery recommendations [16] to prevent inadvertent intramuscular injections [39].
In addition, insulin, pens, and needle prescriptions followed the NHS reimbursement rules and criteria defined by the Italian Drug Agency (AIFA).

The run-in period lasted six months (T-6). At the start, all subjects were trained to perform a structured SMBG and record SyHs and SeHs systematically. Glucose meter recordings were downloaded to the digital CRF platform at 3-month intervals, i.e., at $\mathrm{T}-6, \mathrm{~T}-3$, $\mathrm{T} 0, \mathrm{~T}+3$, and $\mathrm{T}+6$ (see Fig. 2). At T0, all patients were trained in proper IT and were equally randomized $(n=159)$ into either the CG or the IG. The patients in the CG had no further refresher courses until $\mathrm{T}+6$. Conversely, the IG benefited from another structured educational session at $\mathrm{T}+3$ and a monthly phone reminder during the intermediate months $(\mathrm{T}+1, \mathrm{~T}+2, \mathrm{~T}+4, \mathrm{~T}+5$; see Fig. 2). We recorded general biochemistry, HbA1c, hypo frequency and severity, GV, any treatment-related adverse events, and hypo-related hospitalization in the eCRF at $\mathrm{T}-6, \mathrm{~T}$, $\mathrm{T}+3$, and $\mathrm{T}+6$.

\section{Education}

Each general IT education group session involved ten people, lasted approximately $60 \mathrm{~min}$, and employed BD Educational Starter Kits (Becton Dickinson, Inc., Franklin Lakes, NJ, USA), comprising site rotation grids, educational injection technique leaflets, and a bloodglucose log book. The LH "look and feel" teaching method made use of a BD Lipobox, which provides visual and tactile clues for identifying typical LH lesions.

After learning how to rotate injection sites correctly and being instructed not to reuse needles to avoid generating new LHs or worsening existing LHs, all patients received a leaflet with bullet points highlighting the role of IT in optimizing glucose control through unaltered insulin pharmacokinetics and pharmacodynamics [31].

An individualized training session then followed. This addressed real-life problems that influence proper injection pen handling, including cheiroarthropathy, dysphoria, and reduced self-sufficiency. This stage focused on 


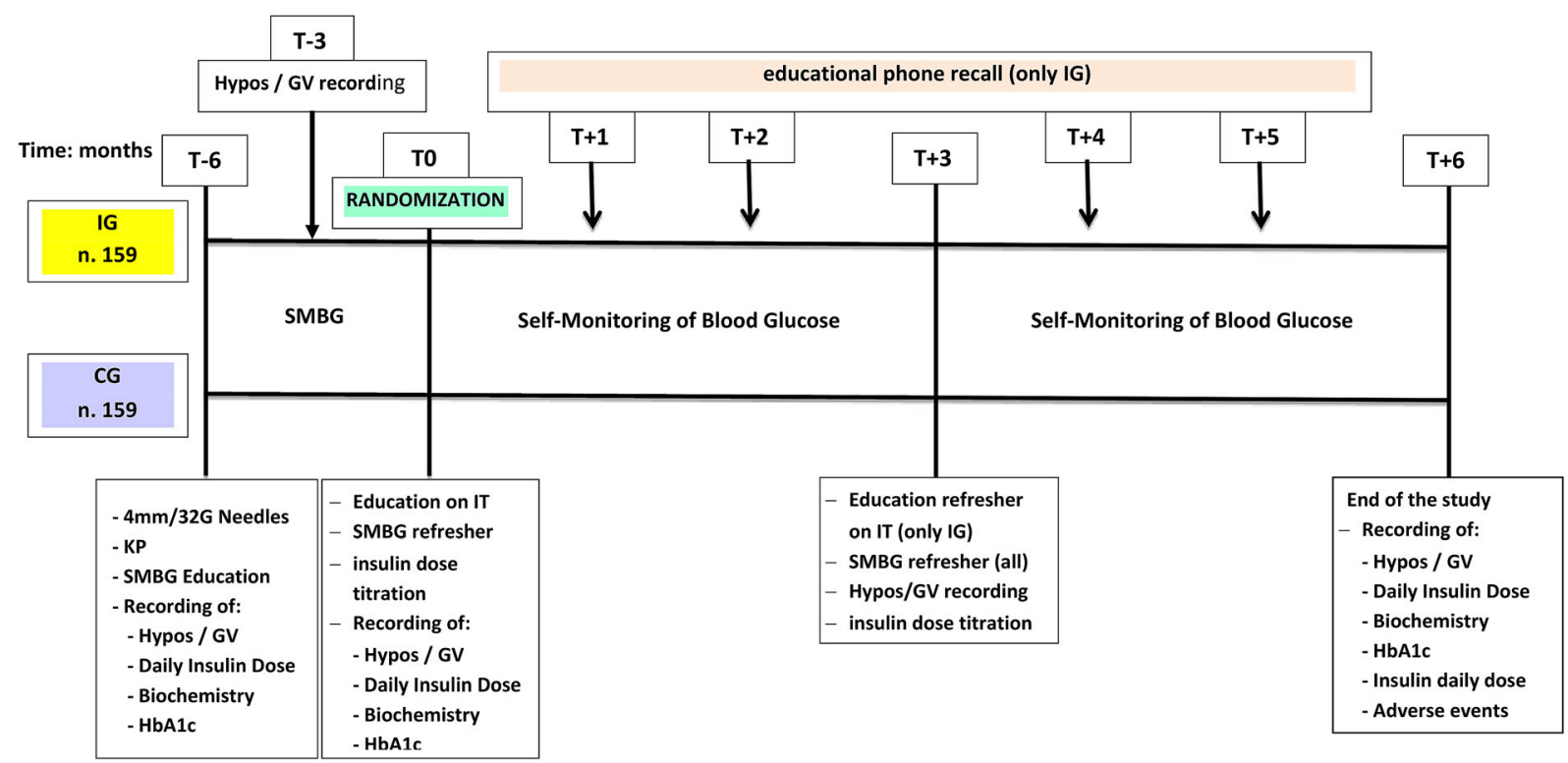

Fig. 2 Schematic of the study protocol. KP KwikPen, Hypos hypoglycemic episodes, $G V$ glycemic variability, $C G$ control group, $I G$ intervention group, $S M B G$ self-monitoring of blood glucose

the relevance of (i) palpating the skin before injection to evade thicker/stiffer sites or LH nodules, (ii) keeping the pen button pressed down for at least $10 \mathrm{~s}$ at the end of the injection, (iii) performing all previously learned IT-related maneuvers autonomously while exploiting any $\mathrm{cm}^{2}$ of healthy skin sequentially, (iv) adjusting the insulin dose as needed, and (v) using the unexplained hypoglycemia and glycemic variation wheel developed by the Forum for Injection Technique (FIT) [40].

Trained HCPs tested participants for their adherence to the three usual insulin titration protocol rules (see below) using a validated questionnaire that included the following four closed answers: (1) no; (2) yes; (3) yes most of the time; (4) no most of the time. Answers (1) and (4) were recorded as "no;" the other two answers were recorded as "yes," as previously described [41].

At $\mathrm{T}-6, \mathrm{~T} 0, \mathrm{~T}+3$, and $\mathrm{T}+6$, all patients performed a full visit, which included a careful injection site examination that allowed the experimenters to populate the eCRF with detailed information on injection habits and hypos collected through a self-administered questionnaire according to a salient, nonintrusive, recent-past-oriented, well-established, and validated procedure $[31,32]$ developed as part of the original Worldwide Injection Technique Questionnaire Study 2016 [42, 43].

\section{Insulin Titration}

As injecting into healthy skin areas leads to faster insulin absorption than injection into LH nodules [13], participants reduced their original DID by $20 \%$ to avoid hypos, as previously recommended [16, 37].

Based on arbitrarily defined criteria, SMBG readings dictated fast-acting and basal insulin dose adjustments as follows: (i) premeal insulin doses were decreased or increased by 2 IU if the corresponding 2-h postprandial glucose levels remained lower than 100 or higher than 140 $\mathrm{mg} / \mathrm{dl}$, respectively, for three consecutive days; (ii) bedtime basal insulin doses were decreased or increased by $2 \mathrm{IU}$ if the corresponding fasting blood glucose values upon waking remained lower than 100 or higher than $180 \mathrm{mg} / \mathrm{dl}$, respectively, for three consecutive days.

\section{Costs}

Healthcare resource utilization, loss of productivity, and other indirect cost items were 
investigated through specific questions, as previously described [24]. The cost calculation utilized institutional and government sources and was performed based on the average NHS reimbursement price list [24]. Examples of prices include: physician home visit $(\mathrm{PHV})=€ 25.82$ [44]; emergency room (ER) visit and treatment $=€ 241.00$ [45]; emergency medical services (EMS) utilization $=€ 128.50$ per hour [46]; working day of family member or caregiver (FMWD and CWD, respectively) $=€ 78.60[47,48]$. The cost of hospitalization (HC), obtained by multiplying the average length of stay (6.7 days) by the average daily hospitalization cost DHC (€750), was calculated as €5.025 [49].

To calculate the cost changes (rather than the actual costs) caused by insulin trapped within LH nodules in our different study groups, we employed the conversion factor $€ 0.02426$ per unit, which was reported by our Spanish colleagues and is-to the best of our knowledge-the only relevant published conversion factor [37].

\section{Statistical Analysis}

An educational intervention study of LHs showed that the HbA1c level decreased by $0.58 \%$ from baseline to the end of follow-up, with a standard deviation of $1.35 \%$ [27]. Thus, by setting the significance level at $\alpha=0.05$ (twosided) and the power at $80 \%$, the minimum sample size for each group was calculated to be 80 patients. Allowing for a $10-15 \%$ dropout rate, the required sample size increased to 90 cases per group. Nevertheless, we enrolled a total of 318 subjects who matched the enrollment criteria.

Patient characteristics are reported here as the mean \pm standard deviation (SD) for continuous variables or the number/percentage for categorical variables.

SyHs and SeHs were expressed as incidence rates (IRs) and 95\% confidence intervals (95\% $\mathrm{CI})$, and were evaluated using the Poisson regression model. Repeated measures analysis of variance (rANOVA) supplemented by the twotailed paired Student's $t$ test with 95\% confidence intervals for parametric variables and Mann-Whitney's $U$ test for nonparametric variables allowed the significance of differences between experimental treatments and times to be explored. The $\chi^{2}$ test with Yates's correction or Fisher's exact test was implemented to achieve categorical variable differentiation.

$p<0.05$ was considered to imply statistical significance. All evaluations were performed using the SAS program (release 9.4, SAS Institute, Cary, NC, USA).

\section{RESULTS}

323 anonymized outpatients who consecutively attended eight DCs for T2DM met the recruitment criteria. Five subjects did not provide their informed consent to participate in the trial, so randomized enrollment involved 318 outpatients with the general characteristics reported in Table 1.55 subjects had end-stage renal disease (ESRD) during the dialysis phase (32.81\%); they were managed in an integrated fashion and followed up in the dialysis units connected to the DCs.

All participants completed the study without reporting any treatment/device-related adverse effects. Good adherence was defined as recording $>80 \%$ of the measured data. Median adherence to SMBG data recording was as high as $92 \%$ (range $84-100 \%$ ), and adherence to hypo recording was also reasonably good. As shown in Table 1, there was no statistically significant difference in general, clinical, and laboratory data or in injection habits or chronic complication rates between the CG and the IG at baseline. However, SyHs and SeHs occurred at relatively high absolute and percentage rates at $\mathrm{T}-6$, and there was no apparent explanation for these high rates of SyHs and SeHs during this period in over $80 \%$ of cases. $9.2 \%$ of participants in the CG and $9.6 \%$ of those in the IG had nighttime SeHs ( $p$ n.s.)

The vast majority of patients presented high $\mathrm{GV}$, poor glycemic control, and vascular complications. DIDs were very similar in the CG and the IG $(0.70 \pm 0.09 \mathrm{IU} / \mathrm{kg} \quad$ bw vs. $0.72 \pm 0.08 \mathrm{IU} / \mathrm{kg} \mathrm{bw}$, respectively; $p$ n.s.). As expected, the CG and the IG showed very 
Table 1 Clinical characteristics and injection habits of patients and controls at enrollment

\begin{tabular}{|c|c|c|}
\hline & $\begin{array}{l}\text { Control group } \\
n=159\end{array}$ & $\begin{array}{l}\text { Intervention group } \\
n=159\end{array}$ \\
\hline Male gender $n(\%)$ & $69(43.40)$ & $71(44.65)$ \\
\hline Age (years) & $63 \pm 12$ & $61 \pm 10$ \\
\hline BMI $\left(\mathrm{kg} / \mathrm{m}^{2}\right)$ & $29.4 \pm 6.2$ & $29.7 \pm 5.7$ \\
\hline HbA1c (\%) & $8.1 \pm 1.1$ & $8.2 \pm 1.2$ \\
\hline DM duration (years) & $11.3 \pm 5.7$ & $11.6 \pm 9.8$ \\
\hline Injections/day $(n)$ & 4 & 4 \\
\hline Insulin treatment duration (years) & $6.7 \pm 7.2$ & $6.5 \pm 9.3$ \\
\hline Daily insulin dose (IU/day) & $56 \pm 12$ & $58 \pm 13$ \\
\hline Number (\%) of patients affected by hypos & $98(61.64)$ & $110(69.18)$ \\
\hline Glycemic variability (mg/dl) & $249 \pm 76$ & $255 \pm 56$ \\
\hline \multicolumn{3}{|l|}{ Injection habits $n(\%)$} \\
\hline Needle reuse $n(\%)$ & $155(97.48)$ & $156(98.11)$ \\
\hline Failure to rotate injection sites $n(\%)$ & $155(97.48)$ & $154(96.86)$ \\
\hline Cold insulin injection $n(\%)$ & $115(72.33)$ & $113(71.07)$ \\
\hline Waiting at end of injection $n(\%)$ & $17(10.69)$ & $16(10.06)$ \\
\hline Drop leakage after injection $n(\%)$ & $155(97.48)$ & $158(99.37)$ \\
\hline Painful injection $n(\%)$ & $13(8.18)$ & $1(0.63)$ \\
\hline Injection into LH nodules $n(\%)$ & $159(100)$ & $159(100)$ \\
\hline \multicolumn{3}{|l|}{ Diabetes complications (\%) } \\
\hline Cardio/cerebrovascular & $28(17.61)$ & $29(17.61)$ \\
\hline Lower limb complications & $13(8.18)$ & $13(8.18)$ \\
\hline Retinopathy & $32(20.13)$ & $33(20.75)$ \\
\hline Nephropathy & $28(17.61)$ & $27(16.98)$ \\
\hline Sensorimotor neuropathy & $18(11.32)$ & $18(11.32)$ \\
\hline Autonomic neuropathy & $9(5.66)$ & $10(6.25)$ \\
\hline
\end{tabular}

The values presented are the mean \pm standard deviation $(M \pm S D)$ or the frequency (\%). There were no significant differences between the control and intervention groups

a Subjects with at least one episode of hypoglycemia in the 4 weeks immediately before randomization; painful injections are defined as those that caused symptoms ranging from slight local discomfort to intense pain

similar improper IT characteristics at $\mathrm{T}-6$, which included many of the mistakes listed below. The most prevalent poor habits were intra-LH injection $(100 \%$ in both the CG and the IG), postinjection drop leakage (97.5 vs. $99.4 \%$ in the CG and the IG, respectively), bleeding (51 vs. 57\% in the CG and the IG, respectively), and ice-cold insulin utilization 


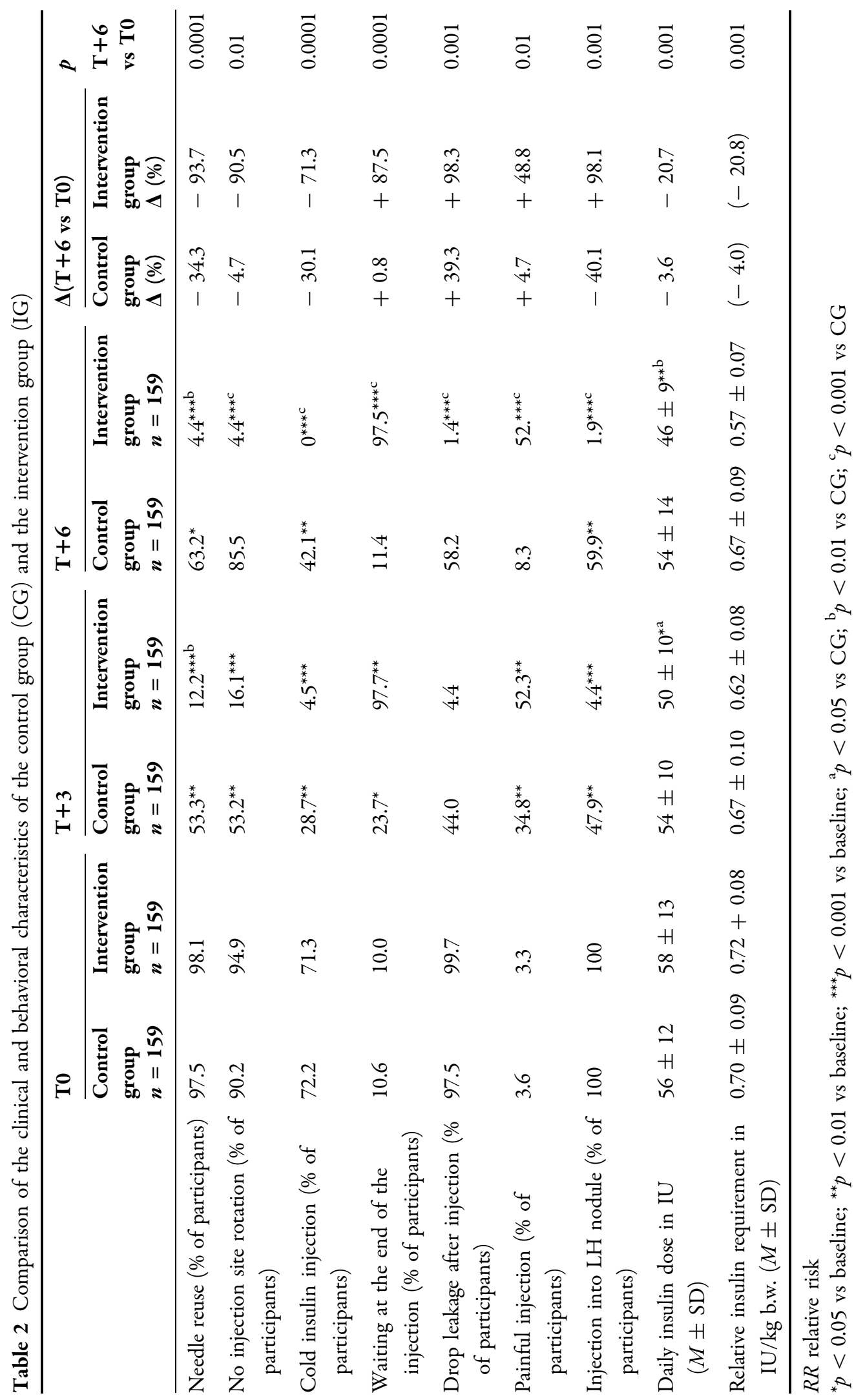




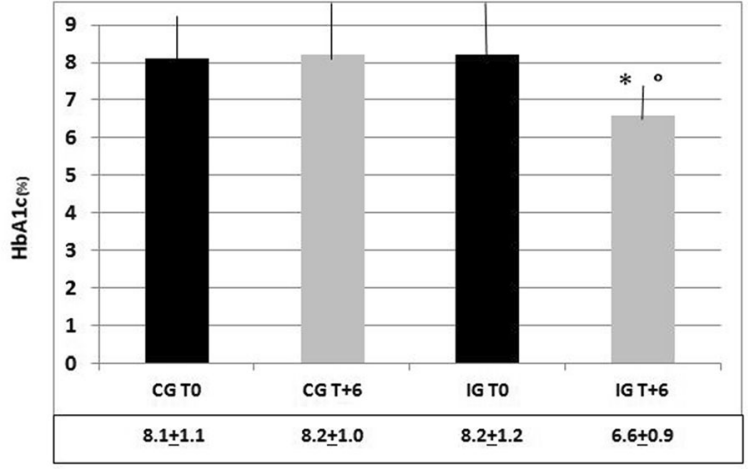

Fig. 3 Mean \pm SD values of HbAlc in the control group (CG) and the intervention group (IG) at T0 and $\mathrm{T}+6$, and significance of the observed differences. ${ }^{*} p<0.01$ vs. IG T0; ${ }^{\circ} p<0.01$ vs. CG T0, and CG T+6

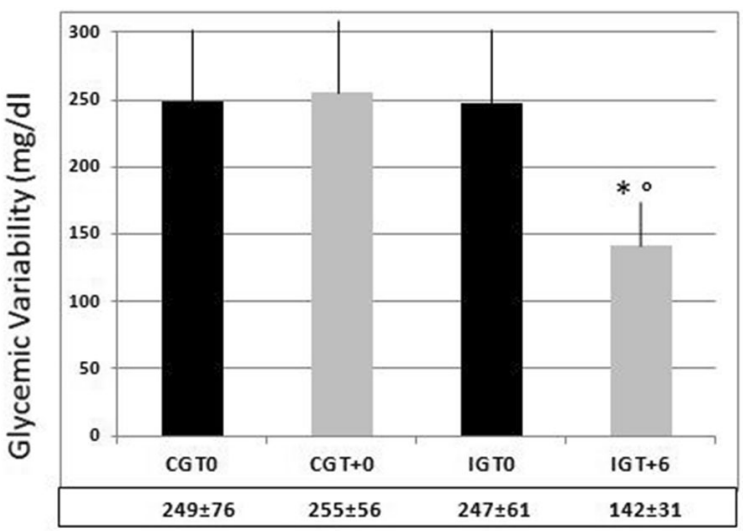

Fig. 4 Mean $\pm S D$ values of glycemic variability $(\mathrm{mg} / \mathrm{dl})$ in the control group (CG) and the intervention group (IG) at T0 and $\mathrm{T}+6$, and significance of the observed differences. ${ }^{*} p<0.01$ vs. IG T0; ${ }^{\circ} p<0.01$ vs. CG T0, and $\mathrm{CG} \mathrm{T}+6$

(72.3 vs. $71.1 \%$ in the $C G$ and the IG, respectively).

However, Table 2 details the progressive changes in injection habits that occurred over time, especially in the IG, which showed a dramatic drop in error rates. It is interesting to note that, in both groups, the number of patients that were still injecting insulin into LHs during the follow-up period was always less than the number who were doing so at baseline $(p<0.001)$, but the statistically significant gap between the CG and the IG in the number of patients that were still injecting insulin into
LHs progressively increased over time (47.9 vs. $4.4 \%$ at $\mathrm{T}+3$ and 59.9 vs. $1.9 \%$ at $\mathrm{T}+6$, respectively; $p<0.001)$.

All other parameters showed a similar trend, including DID, which sharply decreased in the IG vs. both the baseline and the CG $(p<0.0001)$; the DID on the CG remained virtually stable through the study period $(-3.6 \%$ difference vs. baseline; $p$ n.s.). As seen in Table 2 , the DID values at $\mathrm{T}+3$ were almost the same as those at $T+6$ in the $C G$, but these values were significantly different from those observed in the IG.

HbA1c remained almost the same from baseline to $\mathrm{T}+6$ in the CG $(8.1 \pm 1.1 \%$ vs. $8.2 \pm 1.0 \%$, respectively; $p$ n.s.) but decreased significantly in the IG $(8.4 \pm 0.9 \%$ vs. $6.6 \pm 0.9 \%$, respectively; $p<0.01$ ) (Fig. 3). Intermediate levels (i.e., at $\mathrm{T}+3$ ) were $8.0 \pm 1.1$ in the CG and $7.3 \pm 1.1$ in the IG ( $p$ n.s. vs. both baseline and the other group; not shown in Fig. 3). The same behavior was seen for the $\mathrm{GV}$, which barely changed in the CG from T0 to $\mathrm{T}+6 \quad(249 \pm 76 \mathrm{mg} / \mathrm{dl} \quad$ vs. $255 \pm 56 \mathrm{mg} / \mathrm{dl}$, respectively; $p$ n.s.) but sharply decreased in the IG from T0 to T+6 $(247 \pm 61 \mathrm{mg} / \mathrm{dl}$ vs. $142 \pm 31 \mathrm{mg} / \mathrm{dl}$, respectively; $p<0.01$ ) (Fig. 4). At $\mathrm{T}+3$, the GV dropped slightly to $233 \pm 66$ in the CG and to $201 \pm 59$ in the IG as compared to baseline ( $p$ ns vs. baseline and the other group; not shown in Fig. 4).

As shown in Table 3, in the CG, SeH rates throughout the study were statistically indistinguishable: baseline, $16.35 \%$; $\mathrm{T}+3,12.58 \%$; and $\mathrm{T}+6,13.84 \%$. The same was true of the $\mathrm{SyH}$ rates in the CG $(83.65,88.68$, and $84.91 \%$, respectively). Conversely, in the IG, SeH and $\mathrm{SyH}$ rates progressively dropped markedly throughout the study; the rate at each time point was significantly different from the baseline level for the IG and from the corresponding rate at that time point in the CG $(p<0.001)$ (i.e., SeH: $16.35 \%$ at T0, $5.66 \%$ at T+3, $0.63 \%$ at $\mathrm{T}+6$; SyH: $83.65 \%$ at T0, $41.51 \%$ at $\mathrm{T}+3,7.55 \%$ at $\mathrm{T}+6$, respectively). Also, while the baseline and final nighttime hypo rates in the CG were very similar (9.2 and $8.7 \%$, respectively), in the IG the nighttime hypo rate dropped dramatically from $9.6 \%$ to an astonishing $0.0 \%$ at $\mathrm{T}+6$ $(p<0.001$; note that the relevant data are not 
Table 3 Comparison of hypo rates (severe and symptomatic) at different time points $(\mathrm{T} 0, \mathrm{~T}+3$, and $\mathrm{T}+6)$ for the control and intervention groups

\begin{tabular}{|c|c|c|c|c|c|}
\hline & $\begin{array}{l}\text { T0 } \\
n=159\end{array}$ & $\begin{array}{l}\mathrm{T}+3 \\
n=159\end{array}$ & $\begin{array}{l}T+6 \\
n=159\end{array}$ & $\begin{array}{l}\Delta \\
\text { T0 vs } T+6\end{array}$ & $\begin{array}{l}p \\
\text { T0 vs T }+6\end{array}$ \\
\hline \multicolumn{6}{|l|}{ Control group $(n=159)$} \\
\hline Severe hypo $n(\%)$ & $26(16.35)$ & $20(12.58)$ & $22(13.84)$ & $-4(-7.23)$ & n.s. \\
\hline Symptomatic hypo $n(\%)$ & $133(83.65)$ & $141(88.68)$ & $135(84.91)$ & $+2(+1.51)$ & n.s. \\
\hline Severe or symptomatic hypo $n$ & 159 & 159 & 153 & $-6(-3.77)$ & n.s. \\
\hline \multicolumn{6}{|l|}{ Intervention group $(n=159)$} \\
\hline Severe hypo $n(\%)$ & $26(16.35)$ & $9(5.66)^{\mathrm{a}}$ & $1(0.63)^{a, c, d}$ & $-25(-96.15)^{d}$ & 0.0001 \\
\hline Symptomatic hypo $n$ (\%) & $133(83.65)$ & $66(41.51)^{\mathrm{a}, \mathrm{e}}$ & $12(7.55)^{\mathrm{a}, \mathrm{d}}$ & $-121(-90.97)^{\mathrm{d}}$ & 0.0001 \\
\hline Severe or symptomatic hypo $n$ & 159 & $75^{\mathrm{a}, \mathrm{d}}$ & $13^{a, c, d}$ & $-299(-91.82)^{\mathrm{d}}$ & 0.0001 \\
\hline
\end{tabular}

Values shown in the table are the number $(n)$ and percentage (in parentheses) of participants who suffered a hypo

${ }^{\mathrm{a}} p$ vs $\mathrm{T} 0<0.01$

${ }^{\mathrm{b}} p$ vs $\mathrm{T} 0<0.05$

c $p$ vs $\mathrm{T}+3<0.01$

d $p$ vs $C G<0.001$

presented in figures or tables here in order to keep the paper relatively concise).

Table 4 provides details regarding SeH-related costs, which initially were as high as $€ 22,950.2$ in the CG and $€ 28,075.2$ in the IG, but subsequently dropped due to decreases in the hospitalization rate, ER requirement, and HCP/FM help needed. The costs due to SyHswhich were of course much more frequent than SeHs-were also relatively high at T0 ( $€ 14,540.0$ in the CG and €9507.2 in the IG). However, the cost of SyHs remained virtually constant in the CG throughout the follow-up period (costs due to SeHs and SyHs were $€ 24,084.2$ and $€ 15,769.2$, respectively, at the end of the follow-up period), whereas the cost of SyHs dropped dramatically over the course of the study in the IG (costs due to SeHs and SyHs were $€ 25.8$ and $€ 602.5$, respectively, at the end of the follow-up period; $p<0.001$ compared to T0).

The daily insulin dose (DID) was almost the same in the IG and the CG at T0 $(58 \pm 13$ vs. $56 \pm 12$, respectively; $p$ n.s.). However, in the IG, it was significantly lower $(p<0.001)$ at $\mathrm{T}+6$
(46 \pm 9 U, i.e., a $20.7 \%$ decrease at $\mathrm{T}+6$ ) than at baseline, and the DID in the IG was much lower than than that in the CG at $\mathrm{T}+6(54 \pm 14$, i.e., $8 \mathrm{IU}$ lower in the IG on average). Consequently, at the end of follow-up, the daily insulin cost per patient was significantly lower in the IG than in the CG (by €0.02426/ $\mathrm{IU} \times 8 \mathrm{IU}=€ 0.19408$ ). Multiplying 0.194 by 365 days, we find that the education-related insulin cost would decrease by $€ 70.839 /$ patient/ year, or a yearly saving of $€ 70,839$ for every thousand patients who switch to the correct insulin injection technique (see Table S1 in the "Supplementary Information").

\section{DISCUSSION}

Our study clearly shows the superiority of a structured education intervention in which refreshers are performed at regular intervals between scheduled visits as compared to a single initial structured education session in T2DM patients with documented LHs due to incorrect 


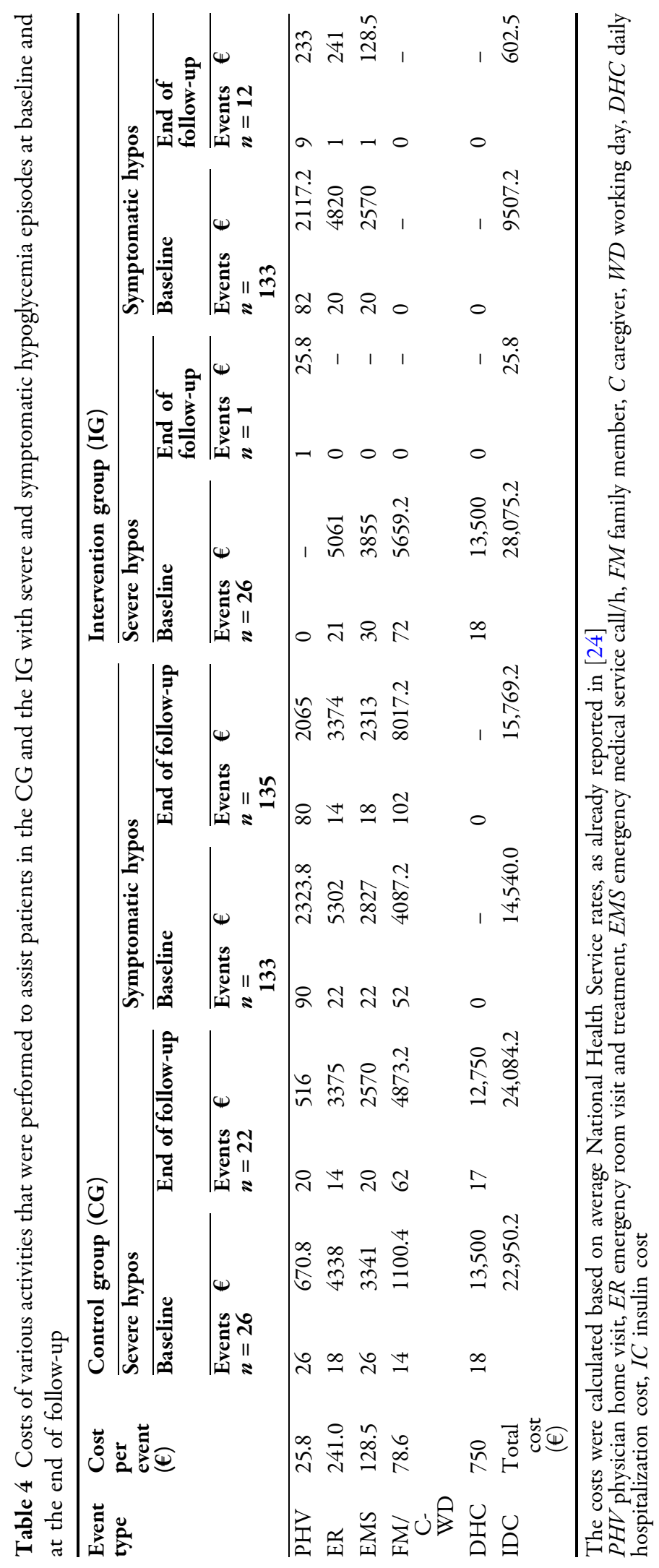


injection technique. This repeated SES-based intervention caused patients to not only recall previous messages but, in particular, to correct previously incorrect elements of injection technique over the course of the study.

In the IG, metabolic control improved significantly in terms of HbA1c level, GV, and number of SyHs and SeHs, and there was a significant reduction in DID over the course of the study. This was obviously accompanied by a dramatic drop in the number of intra-LH injections. No such changes occurred in the CG except for a transient nonsignificant improvement in the abovementioned parameters that occurred shortly after their single SES; this improvement faded over time.

The composite endpoint represented by less frequent hypos (and thus fewer treatment interventions), lower DID, and improved metabolic control led to markedly decreased current health costs and future expenses due to the lower expected complication rate.

A new aspect that emerged from our study was the ability of repeated SESs to eliminate the bad habit of injecting ice-cold insulin. We have already shown that this habit contributes significantly to LH formation but has been largely overlooked in the literature so far [31, 32].

LHs have been the focus of renewed interest from the scientific community in the last few years, as witnessed by the many papers that have recently been published on this topic. However, as easily deduced by consulting the main English-language scientific databases, this interest has not extended beyond admitting that clinicians have not yet been able to curb this phenomenon. We therefore wanted to design a study that would attract clinicians' interest in LHs from a scientific point of view as well as in terms of the practical benefits provided by suitable LH prevention, identification, and treatment strategies. We believed that clinicians would see the advantages of setting up practical, structured education courses that encourage patients to systematically adhere to the correct injection technique if they were proven to decrease the rate of hypos and to improve GV, which would in turn reduce general and health costs relating to the metabolic complications discussed above.
The clinical relevance of the problem addressed in this study was confirmed by a recent rigorous euglycemic clamp study, which showed that insulin injection into LH nodules reduced insulin absorption by about $20-40 \%$ and induced a three- to fivefold increase in within-subject insulin uptake variability (CV\%) compared to injection into healthy adipose tissue [13]. A standardized meal study also showed that intra-LH injections reduce insulin uptake and, not surprisingly, increase postprandial glucose (PPG) levels by a clinically relevant proportion [13]. Despite such clear findings, published research into methods of reducing the prevalence of this phenomenon is limited and does not go beyond current training recommendations aimed at promoting regular site rotation and avoiding needle reuse [12, 47].

LHs-which are extensively reported in the literature-are quite frequent in DM patients, although studies have reported a rather wide range of occurrence rates, suggesting the presence of severe underlying methodological biases [50-53]. This also suggests that, despite the broad dissemination of updated guidelines in the field [12, 50], clinicians may feel more comfortable prescribing drugs than providing correct insulin-handling advice [37]. Many authors have concluded their papers by identifying the need for greater efforts to educate patients in how to suppress LH formation. However, information on the real efficacy and durability of correct injection technique education is limited. A paper published in 2014 [27] focused on the effects of a three-month period of targeted and individualized injection technique training; the study, which involved 346 patients, indicated that the training led to improved glucose control, greater treatment satisfaction, better and more comfortable procedures, and lower required insulin doses.

Moreover, recent intervention studies have proven that proper injection technique training has positive effects on glycemic control and GV in subjects with or without preexisting LHs $[25,27,29,30]$. The first paper, consisting of a preliminary investigation in the form of a brief report, paved the way to (i) a short-duration uncontrolled study, (ii) a pilot trial, and finally (3) another trial that was burdened by 
considerable biases induced by control group contamination or "washing." These studies assessed the effects of correct injection technique education on small groups of patients (i.e., 35, 75, and 95 patients, respectively) for 3-6 months. Thus, the cause-effect relationship that links poor injection practice, LH, and glycemic control has not yet been adequately investigated. Indeed, the few papers that deal with proper injection technique education mostly focus on identifying the most effective element of this education in terms of durable behavioral changes and long-term adherence to correct injection procedures $[29,30]$. This choice reflects the severe time constraints imposed by the long list of patients in the ambulatory setting, which heavily impact any team's ability-regardless of how expert and willing they are-to perform comprehensive injection technique education [31]. On the other hand, nurses who participated in the present study spent much of their time systematically searching for LH nodules and performing LH identification-oriented patient training. Such efforts explain the exemplary achievements attained by the IG, and the results of the study therefore show that complex and multimodal injection technique education is needed to substantially reduce bad injection habits. The approach used in the present work involved two phone calls at regular intervals between two structured interventions occurring at baseline and three months after that. This structured education resulted in improvements in injection technique and caused wrong attitudes to progressively fade away, as demonstrated by the $20 \%$ DID reduction-pointing to healthy skin utilization instead of painless LHs $[16,31,37]$. SeHs were almost entirely abolished and HbA1c levels and GV were significantly reduced. Exposing patients to only a single initial training session-albeit structured, complex, and complete-yielded only partial and transient results. After an initial improvement, which was less prominent than that observed in the IG, almost all of the measured parameters had essentially returned to baseline in the CG by the end of the study. Within the six-month observation period of the present study, intensive structured education was found to yield consistently improved behavior and metabolic results. The far greater avoidance of IT errors during follow-up in the IG than in the CG implies that frequent refresher courses are needed in clinical practice.

Improvements in injection technique were also inferred in this study from a steady reduction in DID reduction, as also reported by other authors $[16,25]$, and from the fact that injections became painful again due to the utilization of healthy skin instead of denervated and mistreated LH-hosting skin [31]. However, further investigation is needed to explore the real durability of the observed effects of proper injection technique education. Our data also suggest that, regardless of how multimodal and intensive it is, a single education session will only provide transient effects; as time goes by, memories of the education session grow fainter, making a return to injection errors more likely. The reasons behind the need for regular top-up education sessions are currently unknown. However, it is plausible that established bad habits can only be eliminated by long-term repeated opposing action.

Furthermore, the substantial effect of introducing periodic injection technique education sessions on the rate of hypos in the IG also has positive impacts on both patient quality of life (data not presented here; quality of life was only explored based on anecdotes from the participants during the follow-up) and the already documented costs of acute hypos and their consequences [18, 25, 29]. Indeed, after our intensive protocol, SeH-related costs dropped dramatically from $€ 28,075.2$ to only $€ 25.8$ in the IG, while SyHs-related costs decreased from $€ 9507.2$ to $€ 602.5$. Both of these cost reductions represent considerable savings.

Even though the cost of a single insulin unit (conventionally set at $€ 0.02426$ ) is minimal, the reduction in DID-related costs achieved via the 6-month injection technique educational program actually represented a notable extra source of savings, as already documented [25, 37]. According to a recent meta-analysis [54], this particular source of savings is nonnegligible considering that $38 \%$ of insulin-treated patients have LH lesions. Given the national prevalence of insulin-treated patients, a saving of $€ 0.02426$ 
per insulin IU-resulting in a reduction of $€ 70.893$ per person per year-would decrease insulin-related costs to the NHS by at least $€ 70,839.20$ per year per 1000 insulin-treated patients who adhere to the correct injection technique due to comprehensive IT education.

Also, according to the UKPDS [52, 53], the complication rate drops significantly with every unit decrease in HbA1c level, so the $1.7 \%$ decrease in HbA1c level (from $8.4 \pm 0.9 \%$ to $6.7 \pm 0.7 \%, p<0.001)$ observed in the IG over the course of the study represents yet another source of savings.

Considering all of the above, besides demonstrating the positive effects of an intensive education program on metabolic control (as also shown by previous studies conducted on smaller populations, e.g., Grassi UK, Francia), our data provide the first evidence that such programs have substantial beneficial economic effects.

\section{Limitations}

Despite being essential to all insulin-treated patients, we understand that not all diabetes units can routinely adopt our training protocol in real-life conditions due to its initial demands on time and human resources. However, based on our experience, we wish to emphasize that HCPs can readily implement this education protocol when staff have been trained to implement it and when it is supported by a well-organized activity timeframe that compensates for the chronic shortage of staff in the NHS.

Another limitation of our study is that it had a relatively short duration, despite the large number of patients involved. However, we expect this limitation to become less of an issue in the future, given that our investigation of the effect of periodically repeated IT education is ongoing.

\section{CONCLUSIONS}

It is regrettable that there is still a relatively high prevalence of LH-affected patients, even though insulin has been available for 100 years and insulin formulations have progressively become more sophisticated, user-friendly, efficacious, and safe. All of these advantages are virtually nullified by the altered insulin pharmacokinetics and pharmacodynamics caused by injection into LH. It is also frustrating that an enormous number of RCTs of insulin preparations are published yearly but there is very little discussion of proper insulin utilization in terms of optimizing the injection technique.

This is the first study to use a real-life multicenter approach to show that comprehensive, repeated education of patients in proper insulin injection technique (i) unequivocally improves their technique and their know-why and knowhow regarding insulin injection and (ii) has inherent economic advantages. We hope to encourage more and more groups to incorporate suitable IT education protocols into their daily routine, as this should improve the quality of the healthcare provided by those groups, enhance patient quality of life, and increase professional satisfaction with patients' achievements.

\section{ACKNOWLEDGEMENTS}

Special thanks are due to Dr. Paola Murano of Nefrocenter Research for providing continuous complimentary logistical support, and to members of AMD-OSDI Study Group on Injection Technique for their editorial assistance and critical manuscript revision. We extend our sincerest thanks to the doctors and nurses of the participating centers. We are also very grateful to the patients for their invaluable and generous participation in the study, as well as for providing their enthusiastic consent to the publication of results arising from the dataset they had contributed to. We also feel deeply indebted to Becton Dickinson Italia S.p.A. (Milan, Italy) for providing educational material with an entirely non-conditioning attitude.

List of Investigators and Collaborators. Members of the Nefrocenter Research and Nyx Start-Up Study Group: Diabetologists: Sandro Gentile, Giuseppina Guarino, Felice 
Strollo, Gerardo Corigliano, Marco Corigliano, Maria Rosaria Improta, Carmine Martino, Antonio Fasolino, Antonio Vetrano, Agostino Vecchiato, Domenica Oliva, Clelia Lamberti, Domenico Cozzolino, Clementina Brancario, Luca Franco. Nutritionist: Teresa Della-Corte. Nephrologists: Maria Luisa Abategiovanna, Maria Amicone, Giovanni Apuzzo, Gennaro Barbuto, Antonio Bassi, Pasquale Boccia, Francesca Borghesi, Alfonso Bosco, Francesco Buono, Tiziana Castellano, Giorgio Chianese, Michele Cicala, Alfonso Ciotta, Secondino Cipriano, Fabrizio G. Crisci, Cristiano Pina, Iris Cupic, Marco De Chiara, Alfonso De Maio, Carlo Del Piano, Chiara Del Prete, Luigi Di Leva, Monica Di Maio, Mauro Di Monte, Alfonso Donnarumma, Enzo Di Stazio, Michele Fabozzi, Maria Gallo, Laura Giordano, Monte Giovanni, Manuela Guerri, Giulia Esposito Iacobitti, Anna Maria La Manna, Gianluca Latte, Fabrizio Lo Iacono, Donato Maietta, Chiara Marano, Maria Federica Finelli, Maria Pasquarella, Mario Acquaviva, Massimo Romano, Gennaro Mattiello, Pietro Miano, Silvia Migliaccio, Alfredo Mignone, Giovanni Monte, Alfredo Fabio Murano, Simona Oliviero, Teresa Pagano, Gabriele Palmentola, Salvatore Postiglione, Pvalo Yavorskiy, Raffaela Esposito, Rosario Reggio, Bruno Riccardi, Eleonora Riccio, Giuseppe Romano, Ersilia Satta, Francesco Antonio Savino, Luisa Scarpati, Domenico Schettino, Giuseppe Spinoso, Erika Troncone, Pasquale Vendemia, Olga Yushkova. Nurses: Paolino Albertini, Lucio Ambrosino, Rosa Vitale Amoroso, Marilena Angrisani, Rosa Apuzzo, Angela Auletta, Fabrizio Barbaro, Gilda Barrella, Alfonso Bartiromo, Maria Battipaglia, Orfeo Belardo, Roberto Bernardo, Valentina Bianco, Pasquale Biondillo, Lucia Bottiglieri, Michela Brida, Orsola Brusco, Giuseppina Buonocore, Zaira Buonocore, Halina Buska, Giuseppe Calabrese, Ida Campolattano, Margherita Capasso, Cecilia Caracciolo, Teresa Carrara, Angela Casaburo, Sara Caso, Marina Assunta Cesarano, Tiziana Cesarini, Annunziata Cherillo, Enrico Cicchella, Michelina Cicchella, Angelo Cimmarosa, Simone Cimmino, Carmelina Cirillo, Pasquale Como, Tiziana Conturso, Giuseppina Cozzolino, Adele Crispino, Raffaele D'Angelo, Salvatore De Felice, Adriano De Filippis,
Margarita De Lucia, Lucia De Micco, Maria Andrea De Vita, Antonio Decostanzo, Carmine D'Elia, Salvatore De Felice, Eligio Della Monica, Angela D'Errico, Veronica D'Esculapio, Marialucia Di Riso, Giovanna Di Maio, Roberta Di Maio, Assunta Di Matola, Assunta Di Nardo, Elisabetta Di Virgilio, Davide Doriano, Eliana Ebraico, Gioacchino Erbaggio, Luca Erpete, Roberta Errichiello, Santo Esca, Gennaro Esposito, Virginia Esposito, Rinaldo Fargnoli, Pasquale Ferrante, Oriana Ferraro, Marco Festinese, Carmen Figlioli, Giovanni Fiorenza, Filomena Fontanella, Michela Fusco, Carmela Gigante, Carmelina Giove, Ruslava Gladka, Anna Guerra, Achille Iannone, Lucia Imbembo, Concetta Imbimbo, Melania Imbimbo, Grazia Indaco, Felice Marco Isola, Antonietta Izzo, Beata Jeschke, Onorina Letizia, Danilo Lettieri, Ana Maria Mandato, Donatella Mannato, Erika Manzi, Lucia Manzo, Carmela Marano, Zuzanna Matusz, Emilio Menna, Sara Milano, Joanna Mlynarska Malgorzata, Carmela Montesarchio, Vincenza Morgillo, Vincenzo Morgillo, Teresa Morrone, Teresa Napolitano, Maria Teresa Natale, Aldo Occhio, Livio Orropesa, Daniela Palmeri, Angela Palmiero, Antonietta Pandolfo, Valentina Passa, Assunta Pastore, Teresa Jadwiga Pazdior, Annamaria Pellino, Elena Petrone, Valentina Pettinati, Filomena Piccolo, Catello Polichetti, Milena Puce, Angela Rainone, Emanuela Repola, Raffaele Riccio, Amelia Ricuperati, Maria Roselli, Enza Ruotolo, Eva Russo, Francesco Russo, Antonietta Salsano, Andrea Schettino, Annalisa Siani, Marilina Siani, Immacolata Silvestri, Pellegrino Spallieri, Annunziata Stasio, Claudia Tabacco, Francesca Tammaro, Maria Emanuela Toscano, Gabriele Ummarino, Federica Variselli, Francesca Vela, Mario Vitale, Paolo Vitale. Members of the AMD-OSDI Study Group: Stefano De-Riu, Nicoletta De-Rosa, Giorgio Grassi, Gabriella Garrapa, Laura Tonutti, Katija Speese, Lia Cucco, MariaTeresa Branca, Amodio Botta.

Funding. No funding or sponsorship was received for this study or the publication of this article. No payment was requested for publication and online posting costs. None of the authors or coworkers received funding or another type of payment for this paper. 
Authorship. All named authors (Sandro Gentile, Giuseppina Guarino, Teresa Della Corte, Giampiero Marino, Ersilia Satta, Carmine Romano, Carmelo Alfarone, Maria Pasquarella, and Felice Strollo) meet the International Committee of Medical Journal Editors (ICMJE) criteria for authorship for this article, take responsibility for the integrity of the work as a whole, and gave their approval for this version to be published.

Authorship Contributions. SG and FS created the paper and wrote it. ES, TDC, GG, GM, $\mathrm{CR}, \mathrm{CA}$, and MP critically read and approved the paper. All have complied with data collection, critically assessed the results, and approved the final text. All collaborators critically read and approved the final text.

Disclosures. Sandro Gentile, Giuseppina Guarino, Teresa Della Corte, Giampiero Marino, Ersilia Satta, Carmine Romano, Carmelo Alfarone, Maria Pasquarella, and Felice Strollo have no financial interests to declare in relation to the present study.

Compliance with Ethics Guidelines. This study was conducted in compliance with good clinical practice standards. The study was led in accordance with the original Declaration of Helsinki and its later amendments, and was approved by the Ethics Committee of Vanvitelli University, Naples, Italy (Trial Registration Number 118-15.04.2018), which served as the central reference EC for all of the diabetes centers involved in this study, being the latter integral part of the same private consortium associated to the abovementioned University. Before enrollment, all of the patients involved in the study provided their written informed consent to both participate in it and to have their data anonymously used for publication.

Data Availability. The datasets analyzed during the present study are available from the corresponding author on reasonable request.

Open Access. This article is licensed under a Creative Commons Attribution-NonCommercial 4.0 International License, which permits any non-commercial use, sharing, adaptation, distribution and reproduction in any medium or format, as long as you give appropriate credit to the original author(s) and the source, provide a link to the Creative Commons licence, and indicate if changes were made. The images or other third party material in this article are included in the article's Creative Commons licence, unless indicated otherwise in a credit line to the material. If material is not included in the article's Creative Commons licence and your intended use is not permitted by statutory regulation or exceeds the permitted use, you will need to obtain permission directly from the copyright holder. To view a copy of this licence, visit http://creativecommons.org/licenses/by$\mathrm{nc} / 4.0 /$.

\section{REFERENCES}

1. Kolendorf K, Bojsen J, Deekert T. Clinical factors influencing the absorption of 125 I-NPH insulin in diabetic patients. Horm Metab Res. 1983;15:274-8.

2. Thow JC, Johnson AB, Fulcher G, Home PD. Different absorption of isophane (NPH) insulin from subcutaneous and intramuscular sites suggests a need to reassess recommended insulin injection technique. Diabet Med. 1990;7:600-2.

3. Thow JC, Home PD. Insulin injection technique: depth of injection is important. Br Med J. 1990;301: 3-4.

4. Engstrom L. Technique of insulin injection: is it important? Pract Diabetes. 1994;11:39.

5. Strauss K, De Gols H, Letondeur C, Matyjaszczyk M, Frid A. The Second Injection Technique Event (SITE), May 2000, Barcelona Spain. Pract Diabetes Int. 2002;19:17-21.

6. Matsumura M, Monden Y, Nakatani T. Improvement of glycemic control by re-education in insulin injection technique. Diabetes. 2007;56(S1):0157OR.

7. Vaag A, Pedersen KD, Lauritzen M, Hildebrandt P, Beck-Nielsen $\mathrm{H}$. Intramuscular verse subcutaneous injection of unmodified insulin: consequences for blood glucose control in patients with type 1 diabetes mellitus. Diabet Med. 1990;7:335-42. 
8. Heinemann L. Variability of insulin absorption and insulin action. Diabetes Technol Ther. 2002;4: 673-82.

9. Frid A, Gunnarsson R, Gunntner P, Linde B. Effects of accidental intramuscular injection on insulin absorption in IDDM. Diabetes Care. 1988;11:41-5.

10. Frid A, Linden B. Where do lean diabetics inject their insulin? A study using computed tomography. Br Med J (Clin Res Ed). 1986;292:1638.

11. Vaag A, Handberg A, Lauritzen M. Variation in absorption of NPH insulin due to intramuscular injection. Diabetes Care. 1990;13(74-6):5.

12. Gentile S, Agrusta M, Guarino G, Carbone L, Cavallaro V, Carucci I, Strollo F. Metabolic consequence of incorrect insulin administration techniques in aging subjects with diabetes. Acta Diabetol. 2011;48:121-5. https://doi.org/10.1007/ s00592-009-0172-x.

13. Famulla S, Hövelmann U, Fischer A, Coester HV, Hermanski L, Kaltheuner M. Insulin injection into lipohypertrophic tissue: blunted and more variable insulin absorption and action and impaired postprandial glucose control. Diabetes Care. 2016;39: $1486 \mathrm{e} 92$.

14. Kreugel G, Beijer HJM, Kerstens MN. Influence of needle size for subcutaneous insulin administration on metabolic control and patient acceptance. Eur Diabetes Nurs. 2007;4:51-5.

15. Hirsch GJ, Gibney MA, Albanese J. Comparative glycemic control, safety and patient ratings for a new $4 \mathrm{~mm} \times 32 \mathrm{G}$ insulin pen needle in adults with diabetes. Curr Med Res Opin. 2010;26:1531-41.

16. Frid AH, Kreugel G, Grassi G, Halimi S, Hicks D, Hirsch LJ, Smith MJ, Wellhoener R, Bode BW, Hirsch IB, Kalra S, Ji L, Strauss KW. New insulin delivery recommendations. Mayo Clin Proc. 2016;91(9):1231-1255. http://creativecommons. org/licenses/by-nc-nd/4.0/

17. Strollo F, Guarino G, Armentano V, on behalf of AMD-OSDI Italian Study Group on Injection Techniques, et al. Unexplained hypoglycaemia and large glycaemic variability: skin lipohypertrophy as a predictive sign. Diabetes Res Open J. 2016;2(1): 24-32. https://doi.org/10.17140/DROJ-2-126.

18. Giorda CB, Ozzello A, Gentile S, Corsi A, Iannarelli $\mathrm{R}$. Incidence and correlates of hypoglycemia in type 2 diabetes. The Hypos-1 study. J Diabetes Metab. 2014;5:344. https://doi.org/10.4172/2155-6156. 1000344.

19. Cryer PE. The barrier of hypoglycemia in diabetes. Diabetes. 2008;57:3169-76.
20. Giorda CB, Rossi MC, Ozzello O, Gentile S, Aglialoro A, Chiambretti A, Baccetti F, Gentile FM, Romeo F, Lucisano G, Nicolucci A, on behalf of the HYPOS-1 Study Group of AMD. Healthcare resource use, direct and indirect costs of hypoglycemia in type 1 and type 2 diabetes, and nationwide projections. Results of the HYPOS-1 study. Nutr Metab Cardiovasc Dis. 2017;27(3):209-16.

21. Goto A, Arah OA, Goto M, Terauchi Y, Noda M. Severe hypoglycaemia and cardiovascular disease: systematic review and meta-analysis with bias analysis. BMJ. 2013;347:f4533.

22. Whitmer RA, Karter AJ, Yaffe K, Quesenberry CP Jr, Selby JV. Hypoglycemic episodes and risk of dementia in older patients with type 2 diabetes mellitus. JAMA. 2009;301:1565-72.

23. Yaffe K, Falvey CM, Hamilton N, Harris TB, Simonsick EM. Association between hypoglycemia and dementia in a biracial cohort of older adults with diabetes mellitus. AMA Intern Med. 2013;173(14):1300-6.

24. Hsu PF, Sung SH, Cheng HM, Yeh JS, Liu WL. Association of clinical symptomatic hypoglycemia with cardiovascular events and total mortality in type 2 diabetes: a nationwide population-based study. Diabetes Care. 2013;36:894-900. https://doi. org/10.1001/jamainternmed.2013.6176.

25. Gentile S, Strollo F, Nefrocenter Research Study Group. Cost saving effects of a short-term educational intervention entailing lower hypoglycaemic event rates in people with type 1 diabetes and lipohypertrophy. Diabetes Res Clin Pract. 2018;143: 320-1. https://doi.org/10.1016/j.diabres.2018.07. 030 (Epub 2018 Aug 1. PMID: 30076872).

26. Liang S, Yin H, Wei C, Xie L, He H, Liu X. Glucose variability for cardiovascular risk factors in type 2 diabetes: a meta-analysis. J Diabetes Metab Disord. 2017;16:45. https://doi.org/10.1186/s40200-0170323-5. https://www.ncbi.nlm.nih.gov/pmc/ articles/PMC5686902/pdf/40200_2017_Article_ 323.pdf. (Epub 2018 Aug 1. PMID: 30076872).

27. Grassi G, Scuntero P, Trepiccioni R, Marubb F, Strauss K. Optimizing insulin injection technique and its effect on blood glucose control. J Clin Transl Endocrinol. 2014;1(4):145-50.

28. Nicolucci A, Cucinotta D, Squatrito S, Lapolla A, Musacchio N, Leotta S, Vitali L, Bulotta A, Nicoziani P, Coronel G, QuoLITy Study Group. Clinical and socio-economic correlates of quality of life and treatment satisfaction in patients with type 2 diabetes. Nutr Metab Cardiovasc Dis. 2009;19(1): $45-53$. 
29. Smith M, Clapham L, Strauss K. UK Lipohypertrophy Interventional Study. Diabetes Res Clin Pract. 2017;126:248-53. https://doi.org/10.1016/j.diabres. 2017.01.020 (Epub 2017 Mar 2 PMID: 28288434).

30. Campinos C, Le Floch JP, Petit C, et al. An effective intervention for diabetic lipohypertrophy: results of a randomized, controlled, prospective multicenter study in France. Diabetes Technol Ther. 2017;19(11):623-32. https://doi.org/10.1089/dia. 2017.0165 (Epub 2017 Oct 23. PMID: 29058477; PMCID: PMC5750448).

31. Gentile S, Guarino G, Corte TD, et al. AMD-OSDI Study Group on Injection Techniques and Nefrocenter Research and Nyx Start-Up Study Group. Insulin-induced skin lipohypertrophy in type 2 diabetes: a multicenter regional survey in Southern Italy. Diabetes Ther. 2020;11(9):2001-17. https:// doi.org/10.1007/s13300-020-00876-0 (Epub 2020 Jul 18. PMID: 32683659; PMCID: PMC7435140).

32. Gentile S, Guarino G, Della Corte T, AMD-OSDI Study Group on Injection Techniques, Nefrocenter Network and Nyx Start-Up Study Group, et al. Lipohypertrophy in elderly insulin-treated patients with type 2 diabetes. Diabetes Ther. 2020. https:// doi.org/10.1007/s13300-020-00954-3 (Epub ahead of print. PMID: 33219928).

33. Gentile S, Strollo F, Satta E, Della Corte T, Romano C, Guarino G, Nefrocenter Research Study Group: Nephrologists, Diabetologists, Nurses. Insulin-related lipohypertrophy in hemodialyzed diabetic people: a multicenter observational study and a methodological approach. Diabetes Ther. 2019;10(4):1423-33. https://doi.org/10.1007/ s13300-019-0650-2 (Epub 2019 Jun 20. PMID: 31222593; PMCID: PMC6612327).

34. Gentile S, Guarino G, Giancaterini A, Guida P, Strollo F, AMD-OSDI Italian Injection Technique Study Group. A suitable palpation technique allows to identify skin lipohypertrophic lesions in insulintreated people with diabetes. Springerplus. 2016;5(5):563. https://doi.org/10.1186/s40064-0161978-y (PMID: 27213130; PMCID: PMC4859222).

35. ADA Classification and Diagnosis of Diabetes. Standards of medical care in diabetes-2019. Diabetes Care. 2019;42(Suppl 1):S13-28. https://doi. org/10.2337/dc19-Sint01.

36. National Center for Health Statistics. International Classification of Diseases, ninth revision, clinical modification (ICD-9-CM). http://www.cdc.gov/ nchs/icd/icd9cm.htm. Accessed Jan 2018.

37. Blanco M, Hernández MT, Strauss KW, Amaya M. Prevalence and risk factors of lipohypertrophy in insulin-injecting patients with diabetes. Diabetes Metab. 2013;39(5):445-53. https://doi.org/10.1016/ j.diabet.2013.05.006 (Epub 2013 Jul 22 PMID: 23886784).

38. Hirsch LJ, Strauss KW. The injection technique factor: what you don't know or teach can make a difference. Clin Diabetes. 2019;37(3):227-33. https://doi.org/10.2337/cd18-0076.

39. Gibney MA, Arce CH, Byron KJ, Hirsch LJ. Skin and subcutaneous adipose layer thickness in adults with diabetes at sites used for insulin injections: implications for needle length recommendations. Curr Med Res Opin. 2010;26(6):1519-30. https://doi.org/ $10.1185 / 03007995.2010 .481203$ 20429833).

(PMID:

40. FIT UK Forum for Injection Technique UK. The UK injectionand infusiontechniquerecommendations,4th ed. 2016. http://www.fit4diabetes.com/ files/4514/7946/3482/FIT_UK_Recommendations_ 4th_Edition.pdf.

41. Gentile S, Ceriello A, Strollo F, on behalf of the AMD START DIAB Study Group. A multicenter Italian survey on diabetes care units reveals a somewhat slow attitude in treatment guideline implementation: are we dealing with therapeutic inertia? Diabetes Res Open J. 2016;2(2):33-44.

42. Frid AH, Hirsch LJ, Menchior AR, Morel DR, Strauss KW. Worldwide injection technique questionnaire study: injecting complications and the role of the professional. Mayo Clin Proc. 2016;91(9):1224-30. https://doi.org/10.1016/j.mayocp.2016.06.012 (PMID: 27594186).

43. Frid AH, Hirsch LJ, Menchior AR, Morel DR, Strauss KW. Worldwide injection technique questionnaire study: population parameters and injection practices. Mayo Clin Proc. 2016;91(9):1212-23. https:// doi.org/10.1016/j.mayocp.2016.06.011 (PMID: 27594185).

44. Council of Italian General Practitioners. Least accepted medical fees. http://www1. ordinemediciroma.it/elenco-completo/ regolamenti/53-tariffaminima-nazionale-deglionorari.html. Accessed 7 Jan 2020.

45. Emergency cost evaluation. http://www.mattoni. salute.gov.it/mattoni/documenti/11_Valutazione_ costi_dell_emergenza.pdf. Accessed 7 Jan 2020.

46. Emergency cost evaluation.http://www.sanita24. ilsole24ore.com/art/regioni-e-aziende/2015-02-12/ emergenza-fiaso-ecco-costi-113448.

php?uuidZAbcvOTBL\&refresh_ceZ1. Accessed 7 Jan 2020.

47. Archives of the Italian Institute of Statistics. http:// www.istat.it/it/archivio/75111. Accessed 7 Jan 2020 . 
48. Demographic balance as recorded in 2013. http:// demo.istat.it/ricostruzione2013/. Accessed 7 Jan 2020 .

49. Ministry of Health, hospitalization cost evaluation. http://www.salute.gov.it/portale/news/p3_2_1_1_1. jsp?menuZnotizie\&pZdalministero\&idZ1411). Accessed 7 July 2020.

50. Gentile S, Grassi G, Armentano V, et al. AMD-OSDI consensus on injection techniques for people with diabetes mellitus. Med Clin Rev. 2016;2:3. https:// medical-clinical-reviews.imedpub.com/ amdosdiconsensus-on-injection-techniques-forpeoplewith-diabetes-mellitus.pdf. Accessed $30 \mathrm{Apr}$ 2020.

51. Gentile S, Strollo F, Guarino G, Diabetologists, Nurses. Why are so huge differences reported in the occurrence rate of skin lipohypertrophy? Does it depend on method defects or on lack of interest? Diabetes Metab Syndr. 2019;13(1):682-6. https:// doi.org/10.1016/j.dsx.2018.11.042 (Epub 2018 Nov 16. PMID: 30641790).
52. Gentile S, Strollo F, Guarino G, on behalf of the AMD-OSDI Italian Injection Technique Study Group, et al. Factors hindering correct identification of unapparent lipohypertrophy. J Diabetes Metab Disord Control. 2016;3(2):42-7. https://doi. org/10.15406/jdmdc.2016.03.00065.

53. Gentile S, Guarino G, Giancaterini A, Guida P, Strollo F, AMD-OSDI Italian Injection Technique Study Group. A suitable palpation technique allows to identify shkin lipohypertrophic lesions in insulin-treated people with diabetes. Springerplus. 2016;5:563. https://doi.org/10.1186/s40064-0161978-y.

54. Deng N, Zhang X, Zhao F, Wang Y, He H. Prevalence of lipohypertrophy in insulin-treated diabetes patients: a systematic review and meta-analysis. J Diabetes Investig. 2017;9(3):536-43. https://doi. org/10.1111/jdi.12742 (Epub ahead of print. PMID: 28862814; PMCID: PMC5934253). 